\title{
A discrete approach for polygonal approximation of irregular noise contours
}

\author{
Phuc Ngo \\ Université de Lorraine, LORIA, UMR 7503, Vandoeuvre-lès-Nancy, F-54506, France \\ hoai-diem-phuc.ngo@loria.fr
}

\begin{abstract}
Polygonal approximation is often involved in many applications of computer vision, image processing and data compression. In this context, we are interested in digital curves extracted from contours of objects contained in digital images. In particular, we propose a fully discrete structure, based on the notion of blurred segments, to study the geometrical features on such curves and apply it in a process of polygonal approximation. The experimental results demonstrate the robustness of the proposed method to local variation and noise on the curve.
\end{abstract}

Keywords: Discrete structure $\cdot$ Polygonal representation $\cdot$ Dominant point

\section{Introduction}

Image processing applications often involve in computing, analyzing and studying objects contained in digital images. In this context, objects are usually extracted by a process of image segmentation. Due to the discrete nature of data to process, contours of these objects are digital curves whose points have integer coordinates. Then, a polygonal approximation of a digital object usually refers to a discrete representation of its contour by a finite number of segments joining two adjacent vertices. It should be mentioned that this discrete modeling of digital objects is required in many applications of computer vision, graphics, image processing and data compression (e.g., [5, 23] ). Furthermore, noise robustness is also an important constraint in the applications.

In the field of digital geometry, new mathematical definitions of basic geometric objects, such as lines or circles, are introduced to better fit these discrete data. In particular, the notion of blurred segment [7, 8] was proposed to deal with digital curves containing noise or other sources of imperfections from the real data by using a fixed thickness parameter $v$. The sequence of blurred segments of thickness $v$ along a digital curve $C$ is called a $v$-tangential cover of $C$ and can be computed in quasi-linear time [6]. It is used in many different contexts to study and analyze the geometrical characteristics of noisy curves such as length, tangent, curvature estimators (e.g., [14,18]).

The present paper aims at designing a fully discrete framework to approximate a digital curve extracted from contour object in a digital image using the notion of blurred segment. More precisely, the proposed method composes of two steps. Firstly, we compute an adaptive tangential cover of the input curve (see Sec. 4). The particularity of this structure is that it contains a sequence of blurred segments with diffrent thicknesses varying in function of noise present along the curve. Such adaptive thicknesses are computed thanks to the local noise estimator of meaningful thickness [11]. Using this structure of adaptive tangential cover, we detect the points of local maximum curvature, 
called dominant points, on the digital curve (see Sec. 57. In brief, dominant points are located in the smallest common zones induced by successive blurred segments. Then, by using a simple measure of angle, we can identify the dominant point as point having the smallest angle. The sequence of dominant points forms a polygonal representation of the curve. However, due to the nature of the tangential cover, dominant points usually stay very close to each others. This is sometimes undesirable as the goal of polygonal approximation is to reduce the amount of necessary information to store a curve, and improve its processing. Therefore, we propose to perform a simplification on the obtained polygon in order to achieve a higher compression while preserving important features of the input curve. The experimental results on both simulated and real data in Sec. 6 show that the proposed method, based on the structure of adaptive tangential cover, allows an effective representation of noisy digital curves.

\section{Background notions}

We recall hereafter some basic notions in the digital geometry literature [12] for analyzing digital curves, particularly the noisy ones.

\subsection{Digital line and blurred segment}

A digital line $D$ is defined as the set of integer points satisfying:

$$
D=\left\{(x, y) \in \mathbb{Z}^{2}: 0 \leq a x+b y+c<\omega\right\}
$$

with $a, b, c \in \mathbb{Z}, \operatorname{gcd}(a, b)=1$, and $\omega \in \mathbb{Z}$ is a constant indicating the thickness of $D$. We denote such a digital line by $D(a, b, c, \omega)$. In particular, when $\omega=\max (|a|,|b|)$ then $D$ is the narrowest 8-connected line and called naif digital line, and $\omega=|a|+|b|$ then $D$ is 4-connected line and called standard digital line. A digital straight segment is a finite subset of $D$. Figure 1 (a-b) show some examples of digital straight lines and segments.

In this work, we consider digital curves, i.e., a finite sequence of discrete points in a predefined order, either counterclockwise or anticlockwise. Such digital curves are generally extracted from contours of objects in digital images.

Definition 1 ([7]). A finite sequence of discrete points $S$ is a blurred segment of thickness $v$ iff (i) $\forall(x, y) \in S,(x, y) \in D(a, b, c, \omega)$, and (ii) the vertical (or horizontal) distance $d=\frac{\omega-1}{\max (|a|,|b|)}$ equals to the vertical (or horizontal) thickness of the convex hull of $S$ such that $d \leq v$.

Let consider a digital curve $C=\left(C_{i}\right)_{0 \leq i \leq n-1}$ of $n$ points. Let $C_{i, j}$ denote the sub-sequence of points from $C_{i}$ to $C_{j}$ in $C$. Let us consider the predicate of a blurred segment of thickness $v$ for $C_{i, j}$ such that

$$
B(i, j, v)=\left\{\begin{array}{l}
\text { True if } C_{i, j} \text { is a blurred segment of thickness } v \\
\text { False otherwise }
\end{array}\right.
$$

Then, we define a maximal blurred segment $(M B S)$ of a sequence of points, not necessarily connected, as follows.

Definition 2 ([7]|). A blurred segment $C_{i, j}$ of $C$ is said to be maximal iff $B(i, j, v), \neg B(i-$ $1, j, v)$ and $\neg B(i, j+1, v)$. 


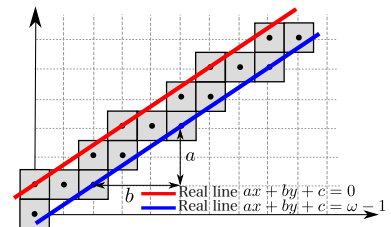

(a)

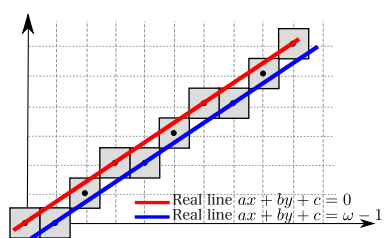

(b)

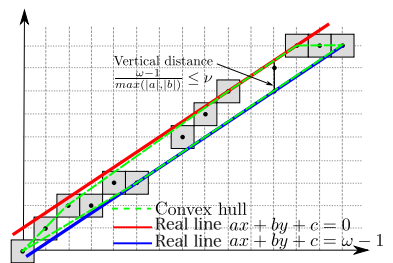

(c)

Fig. 1: Examples of (a) a standard segment belongs to the 4-connected digital line $D(2,-3,3,5)$, (b) a naif segment belongs to the 8-connected digital line $D(2,-3,0,3)$ and (c) a blurred segment of thickness $v=1.5$ belongs to the digital line $D(3,-4,3,7)$.

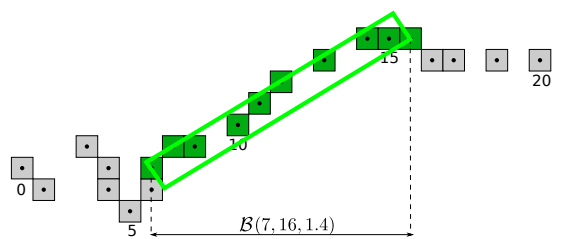

Fig. 2: Maximal blurred segment (in green) $\mathcal{B}(7,16,1.4)$ on a disconnected curve.
The maximal blurred segment $C_{i, j}$ of thickness $v$ is denoted by $\mathcal{B}(i, j, v)$. The blurred segment and maximal blurred segment are respectively illustrated in Fig. 11(c) and Fig. 22 The sequence of maximal blurred segments of a fixed thickness $v$ along $C$ is called $v$-tangential cover of $C$. In [6], an optimal algorithm is proposed to compute this structure with a complexity of $O(n \log n)$ where $n$ is the number of points of $C$.

\subsection{Noise estimator: Meaningful thickness}

Hereafter, we briefly explain a method of noise detection based on the above notions. In [9.10], a notion, called meaningful scale (MS), was proposed to locally estimate the best scale -grid size- to analyze a digital curve. The method is based on the asymptotic properties of the discrete length $L$ of maximal segments.

Theorem 1 ([|13]) Let $\subseteq$ be a simply connected shape in $\mathbb{R}^{2}$ with a smooth $C^{3}$-boundary. Let $p$ be a point on the boundary of $\subseteq$. Let $L^{h}$ be the mean discrete length of maximal segments covering $p$ along the digitized boundary of $\mathfrak{S}$; i.e., $\subseteq \cap h \mathbb{Z} \times h \mathbb{Z}$ (where $h$ represents the grid size). Then, $L^{h}$ is between $\Omega\left(h^{-1 / 3}\right)$ and $O\left(h^{-1 / 2}\right)$ if $p$ is located on a strictly concave or convex part and near $O\left(h^{-1}\right)$ elsewhere.

Roughly speaking, the MS method consists in analyzing each point $p$ on a curve at different grid sizes by sub-sampling and reporting the maximal scale of $p$ for which the mean lengths of maximal segments covering $p$ follow the previous theoretical behavior.

This method of MS estimator has been extended to the detection of meaningful thickness (MT) [11] by using maximal blurred segment primitive together with its thickness parameter. Such a strategy presents an advantage to be easier to implement without any sub-sampling. In particular, the length variation of the maximal blurred segments $\overline{\mathcal{L}}^{t_{i}}$ obtained at different thicknesses $t_{i}$ follows the equivalent properties to the maximal segment defined from sub-sampling. This has been experimentally verified in [11].

Conjecture $1([11])$. The ratio $\mathcal{L}_{j}^{t_{i}} / t^{i}$ in log-scale are approximately affine with negative slopes $s$ located between $-\frac{1}{2}$ and $-\frac{1}{3}$ for a curved part and around -1 for a flat part. 

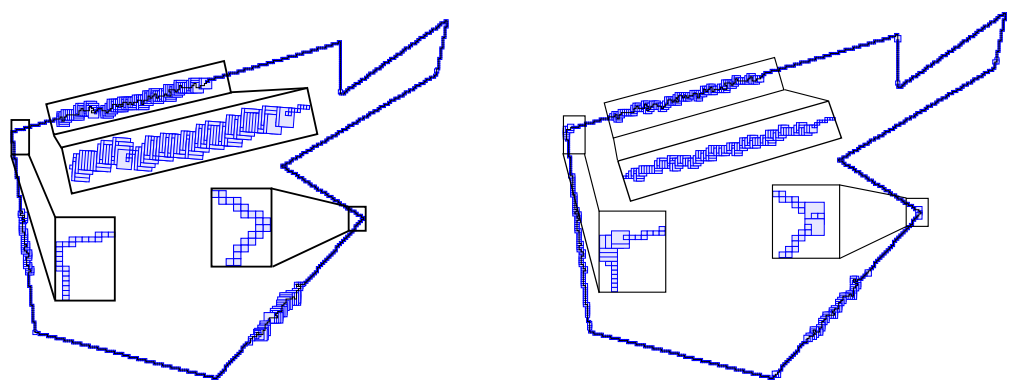

Fig. 3: Comparison between MS (left) and MT (right) estimators. The blue box sizes represent the MS and MT values of each point. Both estimators allow to prevent the local noise perturbation along the curve. However, MT is more sensible at corners than MS.

As suggested in [11], an analysis on the $\log$-scale $\left(\log \left(t_{i}\right), \log \left(\overline{\mathcal{L}}^{t_{i}} / t_{i}\right)\right)_{i=1, \ldots, n}$ at each point $p$ of the contour is performed to determine an indication of local variation at $p$. The graph of log-scale of $p$ is called multi-thickness profile and the first thickness at which the mean length of maximal blurred segments covering $p$ follows the asymptotic behavior is called meaningful thickness of $p$. In other words, the meaningful thickness permits a local estimation of the noise level at each point of a digital contour and provides the thickness of blurred segments should be used to analyze the point. It is worth noting that the MT estimator is slightly more sensitivity than MS at the corners. In particular, high noise level can be detected at corners as illustrated in Fig. 3 . However, MT has the advantage to be able to process non-integer coordinates, non-connected curves, and more efficient in term of computation comparing to MS.

\section{Previous works}

In the digital geomatry field, several attempts have been proposed to handle digital curves containing noise, such as the tangential cover based on maximal blurred segments with a fixed thickness $v$, named $v$-tangential cover [14] (see Fig. 4(c)). Then, the user can manually adjust the parameter $v$ as in [17, 21]. This is however a difficult task to select appropriate thickness. Another solution is to set it as the average of the meaningful thicknesses detected overall the points of the studied curve as in [22]. As the thickness $v$ is globally set for all maximal blurred segments of the $v$-tangential cover, this approach works well when noise is uniformly distributed on the curve, but it is not adapted to local noise. In other words, $v$-tangential cover is inadequate in case of noise varying differently on the curve. To over come this issue, a discrete structure, called adaptive tangential cover (ATC), is presented in [18]. Such a structure is designed to capture the local noise on curve by adjusting the thickness of maximal blurred segments in accordance with the amount of noise present along the curve.

Definition 3 ([|[8] ). Let $C=\left(C_{i}\right)_{0 \leq i \leq n-1}$ be a digital curve. Let $\eta=\left(\eta_{i}\right)_{0 \leq i \leq n-1}$ be the vector of MT associated to each $C_{i}$ of $C$. Let $\mathcal{B}(C)=\left\{\mathcal{B}_{v_{k}}(C)\right\}$ be the set of $v_{k}$-tangential covers for $v_{k} \in \eta$. An adaptive tangential cover (ATC) of $C$ is the sequence of the MBS that $A T C(C)=\left\{\mathcal{B}_{j}=\mathcal{B}\left(B_{j}, E_{j}, v\right) \in \mathcal{B}(C) \mid v=\max \left\{\eta_{t} \mid t \in \llbracket B_{j}, E_{j} \rrbracket\right\}\right\}$ s.t. $\mathcal{B}_{j} \nsubseteq$ $\mathcal{B}_{i}$ for $i \neq j$, where $\llbracket a, b \rrbracket$ is the integer interval between $a$ and $b$, including both. 


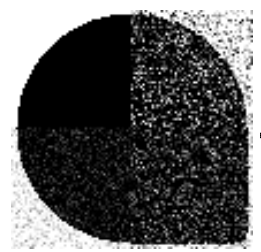

(a)

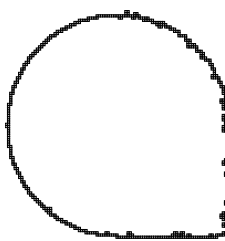

(b)

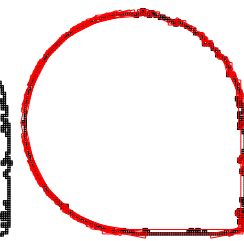

(c)

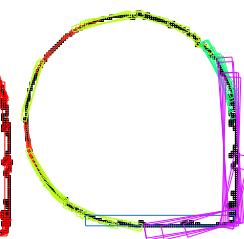

(d)

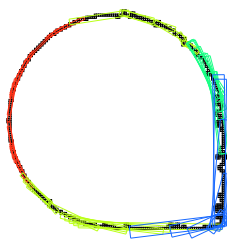

(e)

Fig. 4: Digital contour (b) extracted from noisy image (a) with its 2-tangential cover (c) and its adaptive tangential cover (d) and its modified adaptive tangential cover (e) with MBS of different thickness transmitted from local noise detector of meaningful thickness.

In order to prevent the local perturbation, the MT estimator is integrated in the construction of ATC as a noise detector at each point of the curve. More precisely, the ATC is composed of MBS with thicknesses varying in function of detected perturbations, by the presence of noise or corners, with the MT estimator. In particular, the MBS has bigger thickness at noisy zones, and smaller thickness in zones with less or no noise, as illustrated in Fig. 4(d).

Still in [18], an algorithm is proposed to build the ATC of a given curve based on Def. 3. The algorithm is divided into two steps: (1) labeling the points with thickness values from MT estimator, and (2) building the ATC with the MBS of thickness from the obtained labels. It should be noted that, in the labeling step, the method uses the maximal strategy; i.e., the label associated to the points of a MBS is the maximal meaningful thicknesses detected by MT estimator in the MBS.

The ATC is a good tool for analyzing noisy digital curves [18]. However, when using ACT, we often observe that there are the MBS of big thickness encompassing the significant details of the curve; See Fig. 4(d) and 5(a) as examples of this behavior. This is due to the strategy of labeling the point with the maximal meaningful thickness values. As mentioned in Sec. 2.2 the MT detector is sensible at corners, and provides a less frequent and big thickness at these points. Then, using maximal strategy of meaningful thickness will propagate the improper thicknesses, and generate the big MBS. More importantly, the polygonal representation of a digital curve based on ATC does not fit well the curve, in particular at the corners as illustrated in Fig. 5. a) and (b).

\section{Modified adaptive tangential cover}

As MT estimator may detect a big value of thickness at corners, using the maximal values of meaningful thickness of points in a MBS is not a relevant strategy. To tackle this limitation, we propose a new decision-making of labeling points of the digital curve from the thicknesses detected by the MT estimator.

More precisely, instead of using maximal meaningful thickness, we consider the most frequent thickness for labeling points of MBS constituting the ATC. This is based on the observation that high meaningful thickness at corner is mostly appeared as a singular value while the one caused by noise is a repetitive value. In other words, a more frequent meaningful thickness means a higher probability of presence of noise. 


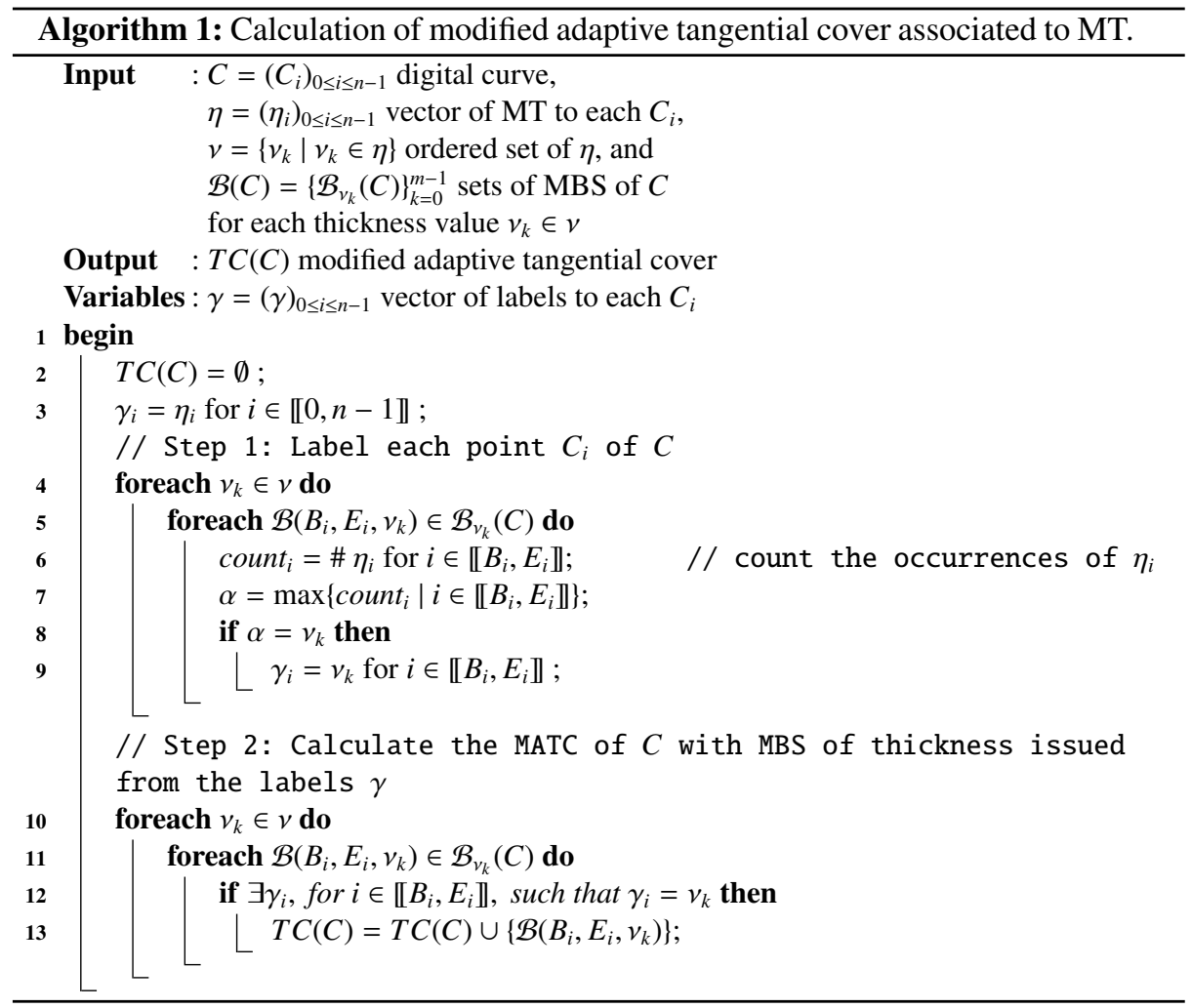

Definition 4. Let $C=\left(C_{i}\right)_{0 \leq i \leq n-1}$ be a digital curve. Let $\eta=\left(\eta_{i}\right)_{0 \leq i \leq n-1}$ be the vector of $M T$ associated to each $C_{i}$ of $C$. Let $\mathcal{B}(C)=\left\{\mathcal{B}_{v_{k}}(C)\right\}$ be the set of $v_{k}$-tangential covers for the different values $v_{k}$ in $\eta$. A modified adaptive tangential cover (MATC) of $C$ is defined as the sequence of the $\operatorname{MBS}\left\{\mathcal{B}_{j}=\mathcal{B}\left(B_{j}, E_{j}, v\right) \in \mathcal{B}(C) \mid v=\max \left\{\operatorname{count}\left(\eta_{t}\right) \mid t \in \llbracket B_{j}, E_{j} \rrbracket\right\}\right\}$ s.t. $\mathcal{B}_{j} \nsubseteq \mathcal{B}_{i}$ for $i \neq j$, where count $\left(\eta_{t}\right)$ is the function that counts the occurrences of $\eta_{t}$ in the vector of MT.

By definition, the MATC possesses the following property.

Property 1. Let $C=\left(C_{i}\right)_{0 \leq i \leq n-1}$ be a digital curve and $\mathcal{B}_{i}=\mathcal{B}\left(B_{i}, E_{i},.\right), \mathcal{B}_{j}=\mathcal{B}\left(B_{j}, E_{j},.\right)$ be two distinct MBS of the modified adaptive tangential cover of $C$. If $i<j$, then $B_{i}<B_{j}$ and $E_{i}<E_{j}$.

From Def. 4, the high thicknesses at corners will not be spread along the curve but only those of noise detected by MT estimator. Thus, the MATC allows to produce a better fitting with MBS along the curve (see Fig. 5.c) and (d)). The principle for computing the MATC is described in Algorithm 1. It is similar to the one in [18] except for the labeling step as described above. It should be mentioned that the computation of MATC has no parameter to set, or only two values related to MT estimator [10]. In particular, the algorithm has $O(k n \log n)$ complexity where $k$ is the number of noise levels detected by MT estimator and $O(n \log n)$ is the complexity for computing $v$-tangential cover [6] of each meaningful thickness detected on a digital curve of $n$ points. In practice, $k$ is much smaller than $n$. 


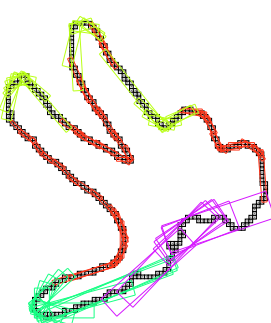

(a)

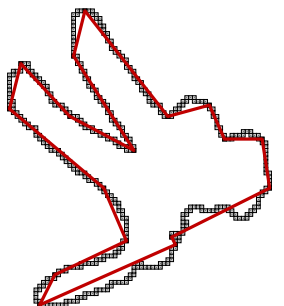

(b)

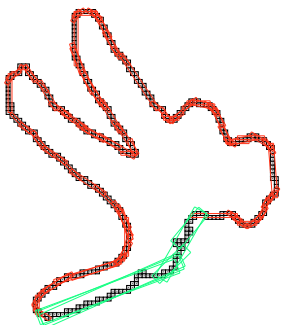

(c)

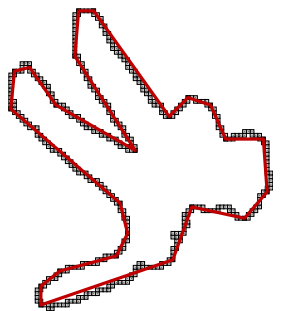

(d)

Fig. 5: Comparison between ATC (a) and MATC (c). The polygonal approximations (red lines) of the contour based on ATC and MATC are respectively (b) and (d).

Figure 5 shows a comparison between the ATC and the MATC on a same digital curve. One can observe that the modified ATC contains of MBS that fit better the shape than those of the former ATC. Furthermore, the polygonal approximation (see Sec. 5) based on the modified ATC archives a more accuracy of the digital curve than this with the ATC. More comparisons are given in Sec.6

\section{Polygonal approximation using MATC}

Polygonal approximation is often involved in many applications of computer vision, graphics, image processing and data compression. In [18, 20], ATC is used as a tool to study digital curves as the sequence of MBS composing ATC provides important information about the geometrical structure of the curve. In particular, it is involed in detecting points of local maximum curvature, called dominant points [3]. Since MATC has the same properties as ATC, we can use the same algorithm in [20] for detecting the dominant point with the proposed structure of MATC.

Let us recall the main idea of the detection method. Firstly, the candidates of dominant points are located in the smallest common zones induced by successive MBS of the tangential cover. Then, a simple measure of angle, as a pseudo-curvature, is estimated for each candidate in the same zone to determine the dominant point in the zone. Such an angle is calculated as the acute angle from the candidate point to the two extremities of the left and right of the MBS composing the zone. Finally, the dominant point is identified as the point with the smallest angle value as the local maximum curvature (see Fig. 6(a)). It should be noticed that, from Prop. 11 the common zone can be detected by simply verifying the extremities of MBS composing the MATC. By joining the straight line segments bounded by two consecutive dominant points, we obtain a polygon $\mathcal{P}$ representing the digital curve. However, due to the nature of the tangential cover, $\mathcal{P}$ usually contains vertices being very close to each others. In the context of polygonal simplification, it is sometimes undesirable as the goal is to reduce the amount of necessary information to store a curve, and improve its processing. Therefore, a reduction on $\mathcal{P}$ is needed to eliminate certain vertices in order to achieve a higher compression of the polygonal approximation and a more effective representation of the original curve. For this, we associate to each vertex of $\mathcal{P}$ a score indicating its importance w.r.t the simplified polygon $\mathcal{P}^{\prime}$. In particular, the score is calculated as the ratio of two factors: 



Fig. 6: Left: Selection of dominant point (in blue) in the smallest common zone (in green) by the three successive MBS. The angle at $C_{9}, C_{1} 0, C_{11}$ and $C_{12}$ are 2.802, 2.733, 2.653 and 2.717 respectively. Then, dominant point is $C_{11}$ which has the smallest angle. Right: Score calculation for dominant points (in red). The point $A$ has a score of $2 / 1.57^{2}=0.812$ which is greater than the point $\mathrm{B}$ of $2.59 / 3.02^{2}=0.284$. Therefore, $\mathrm{A}$ is more important than $\mathrm{B}$.

(1) an error metric of the approximating polygon and the shape and (2) the angular relationship with the two neighboring vertices on $\mathcal{P}$.

More precisely, we would like to remove the nearly collinear vertices of the polygon. Thus, we consider the angle measure of the vertex to its two neighbours on $\mathcal{P}$, and suppress the vertices with big angle measure; i.e., angle closes to $2 \pi$. Besides, we consider the integral sum of square errors (ISSE) as the sum of squared distances of the curve points from the approximating polygon. This error metric evaluates how much the approximated polygon is similar to a curve. In particular, smaller ISSE means better descriptive of the curve by the approximated polygon. For an equivalence of power, the score is computed as $\frac{I S S E}{a n g l e^{2}}$. An illustration is given in Fig. 6(b).

In order to determine the number of dominant points to eliminate during simplification process, we consider the criterion figure of merit, defined as

$$
F O M_{2}=C R^{2} / I S S E
$$

where $C R=n / V$ refers to compression ratio which is defined as the ratio of the number of curve points $n$ and of polygon vertices $V$. It is shown in [4,17] that this function is a performance measure to evaluate the approximation of a curve by a polygon as the compression ratio $(\mathrm{CR})$ describes the effective data reduction, while ISSE describes the discrepancy between the approximating polygon and the curve. In other words, $\mathrm{FOM}_{2}$ with the numerator and the denominator being of equal power represents a compromise between the low approximation error and the benefit of the high data reduction. Therefore, we use $\mathrm{FOM}_{2}$ as objective function in the optimization (maximization) process of polygonal simplification. The algorithm is described in Algorithm 2. Roughly speaking, the polygonal simplification removes incrementally one by one dominant point of highest score until reaching the maximum value of $\mathrm{FOM}_{2}$.

\section{Experimental results and evaluation}

In this section, we present results of the polygonal simplification algorithm using the proposed structure MATC and other recent methods in the literature. In order to evaluate the goodness of a method, we consider the standard evaluation metrics in the literature [16, 24] such as compression ratio (CR), the integral sum of square errors (ISSE), the maximum error $\left(L_{\infty}\right)$ and the figure of merit (FOM, computed by $F O M_{n}=$ $\frac{C R^{n}}{I S S E} \quad$ with $\left.n=1,2,3\right)$. 




\subsection{Polygonal simplification using ATC and MATC}

This section shows the experimental results of the polygonal simplification using the two structures ATC and MATC on the curves with and without noise. The first tests visually compare the results on the curves in Fig. 5] and Fig.7 , and Tab.11 quantifies these results with the evaluation criteria. Further experiments are carried out on KIMA216 database [2]. This database contains 216 shapes, grouped in 18 classes with 12 shapes in each. The quantitative results are shown in Fig. 8 . From the experimental results, we observe a performance improvement of the polygonal simplification using the MATC comparing to the ATC. In particular, the results with MATC provides a better fitting of the curve than those with ATC.

\subsection{Comparisons with other methods}

The comparisons are also performed with two other recent methods whose source code was made publicly available by the authors: Liu et al. [15] and Sivignon [25]. More precisely, the method proposed by Liu et al. in [15] is a polygonal reconstruction from the visual curvature using a parameter $s$ associated to the scale of the contour analysis. The method by Sivignon in [25] computes a simplification of the curve such that the Fréchet distance between the original and the simplified curve is less than the error $e$. 


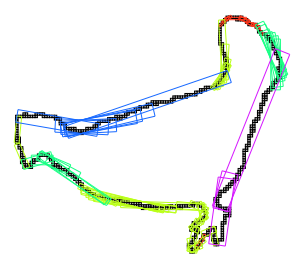

(a)

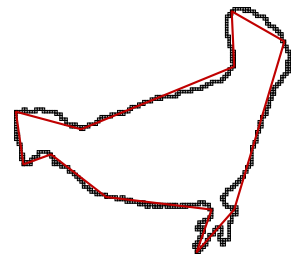

(b)

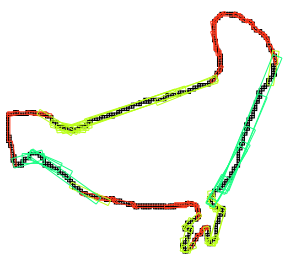

(c)

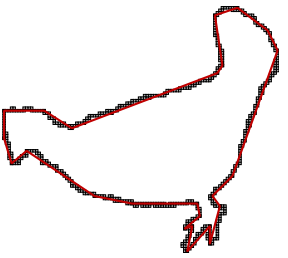

(d)

Fig. 7: Comparison between ATC (a) and MATC (c) on a digital contour. The polygonal approximations of the contour based on ATC (b) and MATC (d).

\begin{tabular}{|c|l|l|l|l|l|l|l|l|}
\hline Curve & Algorithm 2 & $\mathrm{~V} \downarrow$ & $\mathrm{CR} \uparrow$ & ISSE $\downarrow$ & $L_{\infty} \downarrow$ & $F O M \uparrow$ & $F^{\prime} M_{2} \uparrow$ & $F O M_{3} \uparrow$ \\
\hline \hline \multirow{2}{*}{$\begin{array}{c}\text { Fig. } 5 \\
n=454\end{array}$} & ATC & $\mathbf{1 7}$ & $\mathbf{2 6 . 7 0 6}$ & 1047.426 & 4.919 & 0.025 & 0.681 & 18.184 \\
\cline { 2 - 9 } & MATC & 23 & 16.814 & $\mathbf{2 1 3 . 7 6 4}$ & $\mathbf{2 . 3 5 4}$ & $\mathbf{0 . 0 7 1}$ & $\mathbf{1 . 3 9 3}$ & $\mathbf{2 7 . 4 9 1}$ \\
\hline \hline $\begin{array}{c}\text { Fig. } \\
n=548\end{array}$ & ATC & $\mathbf{1 1}$ & $\mathbf{4 9 . 8 1 8}$ & 2280.26 & 6.434 & 0.022 & 1.088 & $\mathbf{5 4 . 2 2 2}$ \\
\cline { 2 - 9 } & MATC & 24 & 22.833 & $\mathbf{4 4 9 . 2 1 4}$ & $\mathbf{2 . 7 1}$ & $\mathbf{0 . 0 5 1}$ & $\mathbf{1 . 1 6 1}$ & 26.501 \\
\hline
\end{tabular}

Table 1: Comparisons of polygonal simplification (Algorithm 2) on the curves in Fig. 5 and Fig. 7]using the structures ATC and MATC.

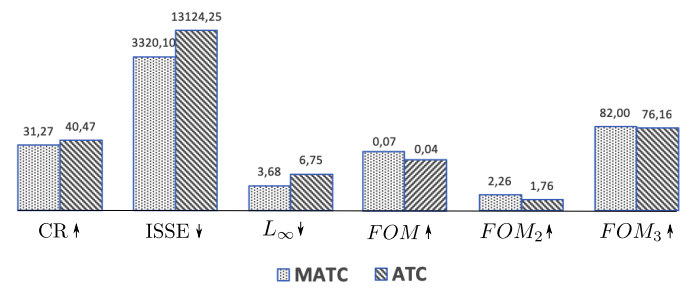

Fig. 8: Comparisons of polygonal simplification (Algorithm 2) on the KIMA216 database [2] using the structures ATC and MATC.

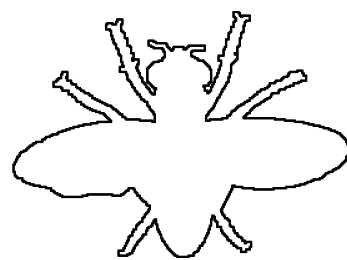

(a)

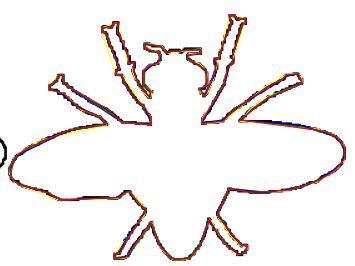

(b)

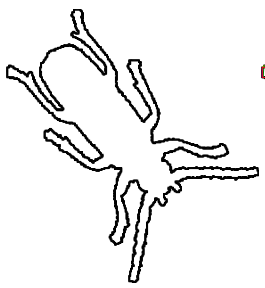

(c)

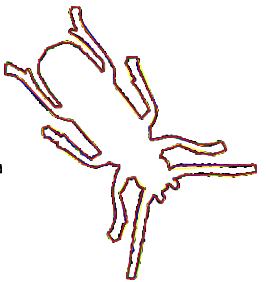

(d)

Fig. 9: (a)-(c): Input curves of polygonal simplification. (b)-(d): The polygonal results using the proposed method (Algorithm 2 with the ATC (in blue) and MATC (in red) structures) and the methods of Sivignon [25] (in green) and Liu et al. [15] (in yellow).

The experiments are performed on the curves in Fig.9 a) and (c). For a fair comparison, we choose the value of scale $s$ in [15] and error $e$ in [25] such that the resulting polygon 


\begin{tabular}{|c|c|c|c|c|c|c|c|c|}
\hline Curve & Methods & $\mathrm{V} \downarrow$ & $\mathrm{CR} \uparrow$ & ISSE $\downarrow$ & $L_{\infty} \downarrow$ & $F O M \uparrow$ & $\mathrm{FOM}_{2} \uparrow$ & $\mathrm{FOM}_{3} \uparrow$ \\
\hline \multirow{4}{*}{$\begin{array}{l}\text { Fig. } 9 \text { a) } \\
n=1990\end{array}$} & Algorithm $2 \mathrm{u}$ & 125 & 15 & 935.088 & 2.575 & 0.016 & 0.241 & 3.609 \\
\hline & Algorithm 2 using MATC & 126 & 15.794 & 931.422 & 2.405 & 0.017 & 0.268 & 4.23 \\
\hline & Liu $[15], s=0.16$ & 124 & 16.048 & 2588.09 & 4.953 & 0.006 & 0.1 & 1.597 \\
\hline & Sivignon $|25|, \mathrm{e}=2.5$ & 124 & 16.048 & 1203.97 & 2.057 & 0.013 & 0.214 & 3.433 \\
\hline \multirow{4}{*}{$\begin{array}{l}\text { Fig.9 (c) } \\
n=2868\end{array}$} & Algorithm 2 using ATC & 127 & 22.583 & 1950.001 & 2.861 & 0.0116 & 0.262 & 5.906 \\
\hline & Algorithm 2 using MATC & 126 & 22.762 & 1924.778 & 3.113 & 0.0118 & 0.269 & 6.127 \\
\hline & Liu $[15], \mathrm{s}=0.021$ & 126 & 22.762 & 5712 & 5.16 & 0.004 & 0.091 & 2.065 \\
\hline & Sivignon $|25|, \mathrm{e}=2.9$ & 128 & 22.406 & 2360.35 & 2.043 & 0.009 & 0.213 & 4.766 \\
\hline
\end{tabular}

Table 2: Comparing results with other methods for the curves in Fig.9 (a) and (c).

has the number of vertices $V$ being closed to this detected by Algorithm 2 using MATC. The results are given in Tab. 2 and Fig. 9(b) and (d).

The proposed method -in particular with MATC- provides, as expected, the best values of $F O M_{n}$ for $n=1,2,3$ but also good values of ISSE. The results by Sivignon's method [25] have the best $L_{\infty}$, it is normal as this method minimizes the distance between the curve and the simplified polygon. Yet, our methods provide competitive results of $L_{\infty}$ as they are very close to the best values.

\section{Conclusion and perspectives}

In this paper, we present the structure of modified adaptive tangential cover (MATC) deduced from the noise estimator of meaningful thickness. The MATC of a digital curve is composed of maximal blurred segments of various thicknesses transmitting the noise levels along the curve and the geometrical structure of the given curve. An algorithm of polygonal simplification is then proposed using this structure of MATC. This parameterfree method permits to obtain good results on the contour of digital shapes with or without noise. The implementation of polygonal simplification, based on the DGtal [1] library, is available for testing from an online demonstration and a GitHub source code repository: http://ipol-geometry.loria.fr/ phuc/ipol_demo/PAMATC_IPOLDemo

The proposed method can be involved in contour-based corner detection which consists in extracting planar curves from images then find local curvature maximal points on the curves. Furthermore, the obtained polygon of the contour of a shape can be applied in a classification process. On the other hand, the MATC can be used to study the geometric estimators of digital curves [18], the decomposition of curves into arcs and segments [19]. In this paper, we only compare the results of the proposed method with two methods [15,25]. In future, we would like to conduct more comparisons with the state-of-the-art methods on the more real data.

\section{References}

1. DGtal: Digital Geometry tools and algorithms library, http://libdgtal.org

2. Kima216 database, http://classif.ai/dataset/kima216

3. Attneave, E.: Some informational aspects of visual perception. Psychological Review 61(3), 183-193 (1954) 
4. Carmona-Poyato, A., Fernández-García, N.L., Medina-Carnicer, R., Madrid-Cuevas, F.J.: Dominant point detection: A new proposal. Image Vis. Comput. 23(13), 1226-1236 (2005)

5. Dilip K., P., Maylor K. H., L.: Polygonal representation of digital curves. In: Digital Image Processing (2012)

6. Faure, A., Buzer, L., Feschet, F.: Tangential cover for thick digital curves. Pattern Recognition 42(10), 2279-2287 (2009)

7. Isabelle, D.R., Fabien, F., Jocelyne, R.D.: Optimal blurred segments decomposition of noisy shapes in linear time. Computers \& Graphics 30(1), 30-36 (2006)

8. Isabelle, D.R., Rémy, J.L., Jocelyne, R.D.: Segmentation of discrete curves into fuzzy segments. Discrete Mathematcis 12, 372-383 (2003)

9. Kerautret, B., Lachaud, J.O.: Multi-scale analysis of discrete contours for unsupervised noise detection. In: International Workshop on Combinatorial Image Analysis. vol. 5852, pp. 187200 (2009)

10. Kerautret, B., Lachaud, J.O.: Meaningful scales detection along digital contours for unsupervised local noise estimation. IEEE Transactions on Pattern Analysis and Machine Intelligence 34(12), 2379-2392 (2012)

11. Kerautret, B., Lachaud, J.O., Said, M.: Meaningful thicknes detection on polygonal curve. In: International Conference on Pattern Recognition Applications and Methods. pp. 372-379 (2012)

12. Klette, R., Rosenfeld, A.: Digital geometry - Geometric methods for digital picture analysis. Morgan Kaufmann (2004)

13. Lachaud, J.O.: Espaces non-euclidiens et analyse d'image : modèles déformables riemanniens et discrets, topologie et géométrie discrète. Habilitation à Diriger des Recherches, Université Bordeaux 1, Talence, France (2006), (in french)

14. Lachaud, J.: Digital shape analysis with maximal segments. In: Workshop on Applications of Discrete Geometry and Mathematical Morphology. pp. 14-27 (2010)

15. Liu, H., Latecki, L.J., Liu, W.: A unified curvature definition for regular, polygonal, and digital planar curves. Inter. Journal Comput. Vision 80(1), 104-124 (2008)

16. Marji, M., Siy, P.: Polygonal representation of digital planar curves through dominant point detection - a nonparametric algorithm. Pattern Recognition 37(11), 2113-2130 (2004)

17. Nasser, H., Ngo, P., Debled-Rennesson, I.: Dominant point detection based on discrete curve structure and applications. Journal of Computer and System Sciences 85, 177-192 (2018)

18. Ngo, P., Debled-Rennesson, I., Kerautret, B., Nasser, H.: Analysis of noisy digital contours with adaptive tangential cover. J. Math. Imaging Vision 59(1), 123-135 (2017)

19. Ngo, P., Nasser, H., Debled Rennesson, I.: A discrete approach for decomposing noisy digital contours into arcs and segments. In: Workshop on Discrete Geometry and Mathematical Morphology for Computer Vision (2016)

20. Ngo, P., Nasser, H., Debled-Rennesson, I.: Efficient dominant point detection based on discrete curve structure. In: International Workshop on Combinatorial Image Analysis. LNCS, vol. 9448 (2015)

21. Nguyen, T.P., Debled-Rennesson, I.: A discrete geometry approach for dominant point detection. Pattern Recognition 44(1), 32-44 (2011)

22. Nguyen, T.P., Kerautret, B., Debled-Rennesson, I., Lachaud, J.: Unsupervised, fast and precise recognition of digital arcs in noisy images. In: International Conference of Computer Vision and Graphics. LNCS, vol. 6374 (2010)

23. Ray, K.S., Ray, B.K.: Polygonal Approximation and Scale-Space Analysis of Closed Digital Curves. CRC Press (2013)

24. Sarkar, D.: A simple algorithm for detection of significant vertices for polygonal approximation of chain-coded curves. Pattern Recognition Letters 14(12), 959-964 (1993)

25. Sivignon, I.: A near-linear time guaranteed algorithm for digital curve simplification under the fréchet distance. Image Processing On Line 4, 116-127 (2014) 